\title{
The Impacts of Hospital Effluent Discharges on the Physico-chemical Water Quality of a Receiving Stream at Ile-Ife, Southwestern Nigeria
}

\author{
O. A. $\mathrm{Ojo}^{1} \&$ I. F. Adeniyi ${ }^{2}$ \\ ${ }^{1}$ Institute of Ecology and Environmental Studies, Obafemi Awolowo University, Ile-Ife, Osun State, Nigeria \\ ${ }^{2}$ Department of Zoology, Obafemi Awolowo University, Ile-Ife, Osun State, Nigeria \\ Correspondence: O. A. Ojo, Institute of Ecology and Environmental Studies, Obafemi Awolowo University, \\ Ile-Ife, Osun State, Nigeria. Tel: 234-0803-454-7242. E-mail: yemmyojo@yahoo.com
}

Received: April 25, 2012 Accepted: October 1, 2012 Online Published: October 29, 2012

doi:10.5539/jsd.v5n11p82 URL: http://dx.doi.org/10.5539/jsd.v5n11p82

\begin{abstract}
In order to contribute to the understanding of the impact of wastewater discharges from a Hospital in south-western Nigeria on the receiving water bodies, the physico-chemical qualities of the two wastewater point sources from the Obafemi Awolowo University Teaching Hospitals Complex (OAUTHC) Ile-Ife were characterized, and their impacts on the water quality of the receiving Elekete stream were assessed. Eight sampling stations were selected for the study: three, were located each on the unimpacted and impacted sections of the receiving Elekete stream while one each was located on the two wastewater point sources from OAUTHC. The physico-chemical parameters investigated include: oxygen parameters, major cations, major anions, nutrient compounds, physical parameters (temperature, turbidity, solids) as well as $\mathrm{pH}$ and conductivity. Samples were collected from each sampling station fortnightly for nine months and analysed using standardised laboratory methods. The results were analysed using relevant statistical methods. The result showed significant difference $(\mathrm{P}<0.05)$ for all parameters between the impacted and unimpacted sections of effluent receiving stream. $\mathrm{SO}_{4}{ }^{2-}$, Total Organic Carbon, $\mathrm{NH}_{4}{ }^{+}, \mathrm{PO}_{4}{ }^{3-}$, and $\mathrm{BOD}_{5}$ were more than three times higher in the impacted section than in the unimpacted section while sample colour, turbidity, total suspended solids, total dissolved solids, total solids, conductivity, alkalinity, acidity, $\mathrm{Ca}^{2+}, \mathrm{Mg}^{2+}, \mathrm{Na}^{+}, \mathrm{K}^{+}, \mathrm{Cl}^{-}, \mathrm{HCO}_{3}^{-}, \mathrm{NO}_{3}^{-}$, and $\mathrm{NO}_{2}^{-}$were about two times higher in the impacted section of the receiving stream than in the unimpacted section. The overall mean concentrations of $293 \mathrm{mg} \mathrm{l}^{-1}$ and $270 \mathrm{mg} \mathrm{l}^{-1} \mathrm{BOD}_{5}$ in the two effluent streams indicate the medium/ strong strength of the wastewater discharges from OAUTHC. This study showed that the wastewater discharge from the (OAUTHC) Ile-Ife has significant impact on the water quality of the receiving streams. The implications are discussed.
\end{abstract}

Keywords: Hospital wastewater, receiving stream, water quality, effluent, physico-chemical parameters, wastewater outfalls

\section{Introduction}

Wastewater generated from hospitals usually contain pathogens, human tissues and fluids, pharmaceuticals, substances with genotoxic properties, chemical substances, heavy metals, and radio-active wastes, which may endanger public health and welfare, and contribute to oxygen demand and nutrient loading of the water bodies and in the process promote toxic algal blooms and leading to a destabilized aquatic ecosystem, if discharged without treatments into water bodies (WHO, 1985; Jackson et al., 1989; NSFC, 1996). Hospital effluents and most public wastewaters eventually end up in streams, rivers, lakes and oceans where they often have deleterious effects on the receiving environment and interfere with the legitimate uses of the water resources e.g. water for drinking, recreation, agricultural and industrial purposes among others. Moreover, these effects are more pronounced and felt in the communities where wastewater treatment is lacking or inadequate.

A typical hospital uses a large volume of water daily and the usage is on the average of 100litres/person/day and the water supply admitted into hospitals varies from 400 to 1200 litres/day/bed (Deloffre-Bornnamour, 1995). In France, the average needs in water of University hospital centre is estimated at 750 litres/bed/day (CCLIN, 1999). This high volume water consumption in high discharge of wastewater is often loaded with micro-organisms, heavy metals, toxic chemicals and radioactive elements emanating from clinical and research laboratories, medical waste incinerators and hospital laundries. As a result, the hospitals generate hybrid wastewater comprising of domestic, industrial and effluents of medical research (Jehannin, 1999; Emmanuel et al., 2001). 
Following the classification of municipal wastewater into strong, medium or weak (based on the concentrations of constituents present) by Melcalf and Eddy (1991), Emmanuel et al. (2001), found out that the values of some physico-chemical parameters of hospital wastewater effluent compare with the medium values of the municipal wastewater. This comparison suggests strong contents of pollutants present in the hospital effluent. Studies have shown that pharmaceutical drugs given to humans and domestic animals which include antibiotics, hormones, strong painkillers, tranquilizers, and chemotherapy chemicals given to cancer patients - are often detected in surface water, in ground water and in drinking water from the tap (Stan \& Linkerharger, 1994). Also, large quantities of drug are excreted by humans and domestic animals, and are distributed into the environment by flushing toilets and by spreading manure and sewage sludge onto and into soil (Montague, 1998). There is ample evidence from research conducted on pharmaceuticals in water bodies, that some sex hormones have potent effects on wild life at concentrations far below $1.0 \mu \mathrm{gl}^{-1}$. For instance, estradiol, the female sex hormone (and a common water pollutant) can alter the sex characteristics of certain fish at concentration of $0.02 \mu \mathrm{gl}^{-1}$ (Raloff, 1998).

While there have been previous studies on wastewater in Ile-Ife area in Nigeria, there is no known study on hospital wastewater effluent. Therefore, in the present study, an assessment of the physico-chemical quality of wastewater from two point sources from the Obafemi Awolowo University Teaching Hospitals Complex (OAUTHC), Ile - Ife, Southwestern Nigeria was undertaken to characterize the physico-chemical quality of the two wastewater point sources at OAUTHC over the period of the study, and to determine the change in the physico-chemical quality of Elekete stream after receiving wastewater effluent from the OAUTHC). This work is expected to provide information on the nature and composition of wastewaters from the OAUTHC, Ile -Ife - a typical hospital complex and the likely impacts of the waste discharge on a receiving freshwater body. The results of the work will also give insight into the recovery pattern of a fresh water body impacted by an hospital effluent. It is also expected that the result of the work will provide information for OAUTHC authority to design and put in place an appropriate wastewater treatment plant and the indicator parameters for monitoring the efficiency of operation of the plant when installed.

\section{Materials and Methods}

\subsection{The Study Area}

This study was carried out at the Ife State Hospital premises of the OAUTHC in Ile-Ife, Osun State Southwestern Nigeria. Ile-Ife lies approximately between latitudes $07^{\circ} 26^{\prime} \mathrm{N}-07^{\circ} 33^{\prime} \mathrm{N}$ of the equator and longitudes $004^{\circ}$ $30^{\prime} \mathrm{E}-004^{\circ} 35^{\prime} \mathrm{E}$ of the Prime Meridian at mean altitude of roughly $300 \pm 50 \mathrm{~m}$ above the mean sea level.

The Ife University Teaching Hospitals Complex (now, known as 0bafemi Awolowo University Teaching hospitals Complex (OAUTHC) was established on the lst November, 1975. Since 1977, the In-patients Admissions were 257,981; Consultant Out-patients Department 1,653,455; General Out-patients Departments 2,335,684, while Accident and Emergency 571,604 and Deliveries 64, 029 (OAUTHC News letter, 2001). OAUTHC Ile- Ife has no functioning wastewater treatment facilities within its premises. There are two wastewater point sources within the hospital premises. The first one is located at the manhole of the sewer conveying wastewater to the abandoned wastewater treatment plant, and the second one is the open gutter of the wastewater from hospital laundry, kitchen and mortuary.

\subsection{Selection and Description of Sampling Stations}

A total of eight (8) sampling stations were selected for this study. These comprise three stations (stations 1, 2 and 3) located on the unimpacted section of the receiving Elekete stream, two stations (stations 4 and 5) on wastewater point sources from the OAUTHC; and the rest three stations (stations 6,7 and 8) on the impacted section of the receiving stream.

Station 1 is located close to the source of Elekete stream. Station 2 is about 510 metres south west of station 1 , and it is a point where people in the local community do their laundry and some other domestic activities. Station 3 is about 400 metres west of station 2, and it is the last station on Elekete stream before the discharge of wastewater into it from the OAUTHC. Station 4 is the manhole of the hospital sewer network close to where wastewater is being discharged directly into Elekete stream. It is about 700 metres south west of station 3 . Station 5 is the open gutter carrying wastewater into Elekete stream from the hospital laundries, mortuary and kitchen. Station 6 is located just below the two outfalls of the hospital wastewater discharge points into Elekete stream, and about 300 metres and 180 metres south west of station 4 and 5 respectively. Station 7 is located about 140 metres south west of station 6 , while station 8 is about 130 metres south west of station 7 . The selection of stations before and after the wastewater outfalls were based largely on accessibility to the receiving stream. 


\subsection{Sampling Programme and Field Determinations}

Samples were taken fortnightly from July 2002 to March 2003. Sampling was carried out from about 09:00. to 15:00 of each sampling date. Each station was sampled about the same time of the day ( $\pm 0.5 \mathrm{hr}$ ) throughout the study period. A total of 128 samples comprising 96 water samples and 32 effluent samples were collected.

Samples for most of analyses (except for Dissolved Oxygen (DO) and Biochemical Oxygen Demand (BOD)) were collected in clean and/or uncontaminated 2.5 litres plastic bottles. Glass reagent bottles were used to collect separate samples for DO and BOD determinations. Samples for DO were fixed on the field immediately after collection using Winkler's reagents (Manganous Sulphate and Alkaline Iodide). Ambient air and water temperatures were measured in-situ using mercury-in-glass bulb thermometer. The sample $\mathrm{pH}$ was measured on the field using a Lovibond $\mathrm{pH}$ comparator with the appropriate disc $(\mathrm{pH} 6.0-7.6)$ and Bromothymol blue indicator solution. Stream transparency was measured using a Secchi disc.

\subsection{Laboratory Analyses}

The analytical determinations of the physico-chemical parameters which include oxygen parameters (dissolved oxygen, and biochemical oxygen demand), major cations, major anions, nutrient compounds (phosphate, nitrate, nitrite, ammonia, silicate and total organic carbon), physical parameters (colour, turbidity, solids) as well as $\mathrm{pH}$ and conductivity of the water/wastewater quality were carried out on the samples collected within the holding time of each parameter, according to the standard method adopted (APHA et al., 1995; DPR, 1991; FEPA, 1991; Gottterman et al., 1978). Sample $\mathrm{pH}$ was measured using a $\mathrm{pH}$ meter with glass electrode (Electronic Instrument Limited, model 7020) while electrical conductivity was measured with a conductivity meter set to give directly in micro siemens per centimeter $\left(\mu \mathrm{Scm}^{-1}\right)$ at $25^{\circ} \mathrm{C}$. The conductivity meter $(\mathrm{K}$ constant $=0.1)$ was standardized from time to time using a set of potassium chloride $(\mathrm{KCl})$ standard solutions (Gottterman et al., 1978).

The Total residue or Total solids (TS) as well as the non filterable residue or Total dissolved solids (TDS) of samples were determined gravimetrically after oven drying them to constant weight at $105 \pm 2^{\circ} \mathrm{C}$ (USEPA, 1983). Total suspended solids (TSS) were calculated as the difference between TS and TDS. Total acidity, Total alkalinity, Total organic carbon (TOC), and Chloride ions $\left(\mathrm{Cl}^{-}\right)$contents were determined by titrimetric methods (Gottterman et al., 1978), Ammonium $\left(\mathrm{NH}_{4}{ }^{+}\right)$, Nitrite $\left(\mathrm{NO}_{2}{ }^{-}\right)$Nitrate $\left(\mathrm{NO}_{3}{ }^{-}\right)$and Sulphate $\left(\mathrm{SO}_{4}{ }^{2-}\right)$ ions were determined by spectrophometric methods. Sodium $\left(\mathrm{Na}^{+}\right)$, Potassium $\left(\mathrm{K}^{+}\right)$were determined by the atomic emission spectrophometric method using a flame analyzer while Silicon, Calcium $\left(\mathrm{Ca}^{2+}\right)$ and Magnesium $\left(\mathrm{Mg}^{2+}\right)$ were determined using a flame atomic absorption spectrophotometer (FAA). The apparent colour of samples was determined on unfiltered samples colorimetrically using a set of Potassium Chloroplatinate- cobalt (Pt-Co.) solutions standards and similarly Turbidity was determined nephelometrically by comparison with turbidity (NTU) standards (APHA et al., 1995).

All the adopted methods have their precision levels in the range of $0.1-10 \%$ and all the recommended quality control (QC) and as well as quality assurance (QA) measures were taken for respective determinations.

\subsection{Statistical Methods of Data Analysis}

The statistical analyses employed in the data analysis include Descriptive statistics (to provide mean, standard deviation) and Student T-test to test degree of differences between data with respect to unimpacted and impacted sampling stations.

\section{Results and Discussion}

The results of the investigated physico-chemical parameters in the sampling stations are presented in Tables 2, 3, 4 and 5. Generally the tables all show a pattern of increase in concentration of the investigated parameters (except DO and $\% \mathrm{O}_{2}$ saturation) in the impacted reach than in unimpacted reach of the receiving stream. Also a general pattern of decrease in concentrations of investigated parameters (except DO and $\% \mathrm{O}_{2}$ saturation which show progressive increase) was observed from the effluent outfall (station 6) to the last sampling station 8 . This confirms the conclusion / suggestion of Chapman (1992) that the effluent would be deemed to impact a stream if the concentration of a given physico-chemical variable is higher in the effluent and decreases significantly with increasing distance downstream.

The overall average value of the total suspended solids (TSS) in the unimpacted reach was $20.94 \mathrm{mgl}^{-1}$, and its value doubled $\left(41.0 \mathrm{mgl}^{-1}\right)$ in the impacted reach of the stream while the overall average value recorded for the effluent sources was $115.0 \mathrm{mgl}^{-1}$ as shown in Table 1. The recorded value of $41.0 \mathrm{mgl}^{-1}$ in the impacted receiving stream is higher than the Nigerian Federal Environmental Protection Agency (FEPA) stipulated $30.0 \mathrm{mgl}^{-1} \mathrm{TSS}^{-}$ limit for discharge into the surface water and this can result into the aesthetic degradation of the receiving stream and other effects such as the reduction in the light penetration through the water body and its attendant effect on 
the photosynthetic rates of algae and submerged macrophytes and clogging of respiratory surfaces of gills of fish or nets of filter feeding invertebrates (Hellawell, 1986). Emmanuel et al. (2001) and Guatam et al. (2007) carried out similar studies on hospital effluent, and recorded an average of $225 \mathrm{mgl}^{-1}$ and $531 \mathrm{mgl}^{-1}$ of TSS respectively, but in this study TSS ranged between 102 and $155 \mathrm{mgl}^{-1}$ in the hospital sewers stream and 70 to $143 \mathrm{mgl}^{-1}$ in the open gutter stream, and according to Metcalf and Eddy (1991), the effluents can be classified as weak effluents in terms of its total suspended solids.

Table 1. Overall mean values of the investigated hydro - physical water quality parameters of receiving stream and effluent samples

\begin{tabular}{|c|c|c|c|c|c|}
\hline \multirow{2}{*}{ Water Body } & \multirow{2}{*}{ Station } & Water Temp. & Colour & Turbidity & TSS \\
\hline & & $\left({ }^{\circ} \mathrm{C}\right)$ & (Pt- Co.) & (NTU) & $\left(\mathrm{mgl}^{-1}\right)$ \\
\hline \multirow{6}{*}{$\begin{array}{c}\text { Unimpacted stream } \\
\text { reach }\end{array}$} & \multirow{2}{*}{1} & 26.2 & 48.9 & 11.5 & 8.8 \\
\hline & & $( \pm 1.5)$ & $( \pm 29)$ & $( \pm 6.2)$ & $( \pm 3.5)$ \\
\hline & \multirow{2}{*}{2} & 24.5 & 133.9 & 31.6 & 20.8 \\
\hline & & $( \pm 1.9)$ & $( \pm 43.6)$ & $( \pm 10)$ & $( \pm 10.4)$ \\
\hline & \multirow{2}{*}{3} & 24.8 & 77 & 18.8 & 33.3 \\
\hline & & $( \pm 2.0)$ & $( \pm 45.2)$ & $( \pm 10.1)$ & $( \pm 16.4)$ \\
\hline \multirow{2}{*}{\multicolumn{2}{|c|}{ Overall mean $( \pm \mathrm{SD})$}} & 25.49 & 86.58 & 20.60 & 20.94 \\
\hline & & $( \pm 1.97)$ & $( \pm 53.91)$ & $( \pm 12.35)$ & $( \pm 15.33)$ \\
\hline \multirow{4}{*}{ Effluent discharges } & \multirow{2}{*}{4} & 25.8 & 530.3 & 125.8 & 129.6 \\
\hline & & $( \pm 1.2)$ & $( \pm 236.3)$ & $( \pm 57.8)$ & $( \pm 26.8)$ \\
\hline & \multirow[b]{2}{*}{5} & 26.9 & 368.7 & 84.8 & 100.5 \\
\hline & & $( \pm 1.8)$ & $( \pm 291.4)$ & $( \pm 65.8)$ & $( \pm 24.8)$ \\
\hline \multirow{2}{*}{\multicolumn{2}{|c|}{ Overall mean $( \pm$ SD) }} & 26.33 & 451.47 & 105.22 & 115.03 \\
\hline & & $( \pm 1.67)$ & $( \pm 283.25)$ & $( \pm 66.34)$ & $( \pm 30.18)$ \\
\hline \multirow{6}{*}{$\begin{array}{l}\text { Impacted stream } \\
\text { reach }\end{array}$} & \multirow{2}{*}{6} & 25.1 & 179.3 & 43.1 & 62.2 \\
\hline & & $( \pm 1.9)$ & $( \pm 65.3)$ & $( \pm 14.8)$ & $( \pm 15)$ \\
\hline & \multirow{2}{*}{7} & 24.9 & 104 & 26.4 & 33.8 \\
\hline & & $( \pm 1.4)$ & $( \pm 41.7)$ & $( \pm 11.5)$ & $( \pm 8.8)$ \\
\hline & \multirow{2}{*}{8} & 24.9 & 64.2 & 17.6 & 27.1 \\
\hline & & $( \pm 1.5)$ & $( \pm 36.1)$ & $( \pm 7.6)$ & $( \pm 5.3)$ \\
\hline \multirow{2}{*}{\multicolumn{2}{|c|}{ Overall mean $( \pm$ SD) }} & 25.00 & 113.58 & 29.04 & 41.0 \\
\hline & & $( \pm 1.64)$ & $( \pm 70.99)$ & $( \pm 15.94)$ & $( \pm 18.69)$ \\
\hline
\end{tabular}


Table 2. The overall mean concentrations of the investigated general chemical water quality parameters of the receiving stream and effluent samples

\begin{tabular}{|c|c|c|c|c|c|c|c|}
\hline \multirow{2}{*}{ Water Body } & \multirow{2}{*}{ Station } & \multirow{2}{*}{ pH } & \multirow{2}{*}{$\begin{array}{c}\text { Conductivity } \\
(\boldsymbol{\mu S} / \mathbf{c m})\end{array}$} & \multirow{2}{*}{$\begin{array}{c}\text { TDS } \\
\left(\mathrm{mgl}^{-1}\right)\end{array}$} & \multirow{2}{*}{$\begin{array}{c}\mathrm{TS} \\
\left(\mathrm{mgl}^{-1}\right)\end{array}$} & \multirow{2}{*}{$\begin{array}{c}\text { Alkalinity } \\
\left(\mathrm{mg} \mathrm{CaCO}_{3} \mathrm{I}^{-1}\right)\end{array}$} & \multirow{2}{*}{$\begin{array}{c}\text { Acidity } \\
\left(\mathrm{mg} \mathrm{CaCO}_{3} \mathrm{I}^{-1}\right)\end{array}$} \\
\hline & & & & & & & \\
\hline \multirow{6}{*}{$\begin{array}{c}\text { Unimpacted } \\
\text { Stream } \\
\text { Reach }\end{array}$} & & 6.74 & 132 & 94.4 & 103.2 & 55.93 & 20.8 \\
\hline & 1 & $( \pm 0.12)$ & $( \pm 7.0)$ & $( \pm 6)$ & $( \pm 5)$ & $( \pm 7.02)$ & $( \pm 9.4)$ \\
\hline & & 6.8 & 110 & 81.4 & 102.1 & 54.6 & 21.3 \\
\hline & 2 & $( \pm 0.15)$ & $( \pm 12)$ & $( \pm 9.4)$ & $( \pm 10.5)$ & $( \pm 5.8)$ & $( \pm 8.6)$ \\
\hline & & 7.14 & $165(+13)$ & 113.8 & 147.4 & 79.3 & 16.4 \\
\hline & 3 & $( \pm 0.19)$ & $165( \pm 13)$ & $( \pm 11.4)$ & $( \pm 18.8)$ & $( \pm 6.8)$ & $( \pm 4.3)$ \\
\hline \multirow{2}{*}{\multicolumn{2}{|c|}{ Overall mean $( \pm$ SD) }} & 6.88 & 135.63 & 96.52 & 117.56 & 63.29 & 19.47 \\
\hline & & $( \pm 0.24)$ & $( \pm 25.6)$ & $( \pm 16.38)$ & $( \pm 24.92)$ & $( \pm 13.19)$ & $( \pm 8.19)$ \\
\hline \multirow{4}{*}{$\begin{array}{c}\text { Effluent } \\
\text { discharges }\end{array}$} & 4 & 7.2 & 828 & 552.4 & 682.1 & 350.6 & 123.2 \\
\hline & 4 & $( \pm 0.23)$ & $( \pm 283)$ & $( \pm 185.2)$ & $( \pm 190.7)$ & $( \pm 107.4)$ & $( \pm 45.9)$ \\
\hline & 5 & 6.7 & 534 & 327.6 & 428.1 & 191.3 & 83.6 \\
\hline & 5 & $( \pm 0.35)$ & $( \pm 162)$ & $( \pm 93.7)$ & $( \pm 105.6)$ & $( \pm 44.2)$ & $( \pm 46.3)$ \\
\hline \multirow{2}{*}{$\begin{array}{c}\text { Overall } \\
\text { mean }( \pm \text { SD })\end{array}$} & & 6.69 & 681.0 & 440.0 & 555.06 & 270.93 & 103.44 \\
\hline & & $( \pm 0.36)$ & $( \pm 277.7)$ & $( \pm 187.84)$ & $( \pm 202.9)$ & $( \pm 116.26)$ & $( \pm 51.04)$ \\
\hline \multirow{6}{*}{$\begin{array}{c}\text { Impacted } \\
\text { Stream Reach }\end{array}$} & ( & 6.88 & 236 & 168.6 & 230.8 & 114.8 & 35.2 \\
\hline & 0 & $( \pm 0.15)$ & $( \pm 91)$ & $( \pm 59.8)$ & $( \pm 69.4)$ & $( \pm 37.1)$ & $( \pm 19.8)$ \\
\hline & 7 & 7.08 & 207 & 145.5 & 179.2 & 101.1 & 25.2 \\
\hline & 1 & $( \pm 0.2)$ & $( \pm 38)$ & $( \pm 29.8)$ & $( \pm 28.3)$ & $( \pm 25.1)$ & $( \pm 14.6)$ \\
\hline & & 7.04 & 212 & 137.6 & 164.7 & 95.62 & 24.7 \\
\hline & 8 & $( \pm 0.18)$ & $( \pm 50)$ & $( \pm 29.2)$ & $( \pm 26.5)$ & $( \pm 25.2)$ & $( \pm 13.6)$ \\
\hline \multirow{2}{*}{\multicolumn{2}{|c|}{ Overall mean $( \pm$ SD) }} & 7.0 & 219.81 & 150.56 & 191.56 & 104.31 & 28.35 \\
\hline & & $( \pm 0.20)$ & $( \pm 67.28)$ & $( \pm 44.57)$ & $( \pm 54.5)$ & $( \pm 30.93)$ & $( \pm 17.12)$ \\
\hline
\end{tabular}


Table 3. The overall mean concentrations of the investigated major ions of the receiving stream and effluent samples

\begin{tabular}{|c|c|c|c|c|c|c|c|c|}
\hline \multirow{2}{*}{ Water body } & \multirow{2}{*}{ Station } & $\begin{array}{c}\text { Calcium } \\
\left(\mathrm{Ca}^{2+}\right)\end{array}$ & $\begin{array}{c}\text { Magnesium } \\
\left(\mathrm{Mg}^{2+}\right)\end{array}$ & $\begin{array}{c}\text { Sodium } \\
\left(\mathrm{Na}^{+}\right)\end{array}$ & $\begin{array}{c}\text { Potassium } \\
\left(\mathrm{K}^{+}\right)\end{array}$ & $\begin{array}{c}\text { Chloride } \\
\left(\mathrm{Cl}^{-}\right)\end{array}$ & $\begin{array}{l}\text { Sulphate } \\
\left(\mathrm{SO}_{4}{ }^{2-}\right)\end{array}$ & $\begin{array}{c}\text { Bicarbonate } \\
\left(\mathrm{HCO}_{3}^{-}\right)\end{array}$ \\
\hline & & \multicolumn{7}{|c|}{$\left(\mathrm{mgl}^{-1}\right)$} \\
\hline \multirow{6}{*}{$\begin{array}{c}\text { Unimpacted } \\
\text { Stream } \\
\text { Reach }\end{array}$} & \multirow{2}{*}{1} & 13.9 & 4.6 & 4.1 & 2.2 & 11.3 & 0.7 & 69.4 \\
\hline & & $( \pm 1.7)$ & $( \pm 1.1)$ & $( \pm 1.2)$ & $( \pm 0.3)$ & $( \pm 0.97)$ & $( \pm 0.5)$ & $( \pm 7.8)$ \\
\hline & \multirow{2}{*}{2} & 12.5 & 4.5 & 4.2 & 2.5 & 9.3 & 0.9 & 66.7 \\
\hline & & $( \pm 1.7)$ & $( \pm 1.1)$ & $( \pm 1.1)$ & $( \pm 0.6)$ & $( \pm 1.6)$ & $( \pm 0.7)$ & $( \pm 7.1)$ \\
\hline & \multirow{2}{*}{3} & 17.2 & 5.7 & 6.2 & 3.2 & 11.6 & 0.8 & 96.8 \\
\hline & & $( \pm 1.4)$ & $( \pm 2.0)$ & $( \pm 1.1)$ & $( \pm 0.6)$ & $( \pm 1.3)$ & $( \pm 0.6)$ & $( \pm 8.3)$ \\
\hline \multirow{2}{*}{$\begin{array}{c}\text { Overall } \\
\text { mean }( \pm \text { SD })\end{array}$} & & 14.57 & 4.92 & 4.82 & 2.64 & 10.74( & 0.80 & 38.14 \\
\hline & & $( \pm 2.62)$ & $( \pm 1.60)$ & $( \pm 1.4)$ & $( \pm 0.68)$ & $\pm 1.71)$ & $( \pm 0.65)$ & $( \pm 7.73)$ \\
\hline \multirow{4}{*}{$\begin{array}{c}\text { Effluent } \\
\text { discharges }\end{array}$} & \multirow{2}{*}{4} & 59.3 & 14.4 & 18.5 & 8.4 & 76.3 & 43.4 & 427.7 \\
\hline & & $( \pm 14)$ & $( \pm 7.5)$ & $( \pm 2.4)$ & $( \pm 1.3)$ & $( \pm 37)$ & $( \pm 13.4)$ & $( \pm 131.3)$ \\
\hline & \multirow{2}{*}{5} & 41.4 & 11.2 & 15 & 6.7 & 47.1 & 21.3 & 233.3 \\
\hline & & $( \pm 12.6)$ & $( \pm 5.3)$ & $( \pm 4.5)$ & $( \pm 1.7)$ & $( \pm 14.3)$ & $( \pm 10)$ & $( \pm 54.1)$ \\
\hline \multirow{2}{*}{$\begin{array}{c}\text { Overall } \\
\text { mean }( \pm \text { SD })\end{array}$} & & 50.37 & 12.36 & 16.70 & 7.59 & 52.86 & 30.29 & 163.43 \\
\hline & & $( \pm 16.29)$ & $( \pm 6.12)$ & $( \pm 4.05)$ & $( \pm 1.75)$ & $( \pm 22.76)$ & $( \pm 13.29)$ & $( \pm 69.86)$ \\
\hline \multirow{6}{*}{$\begin{array}{l}\text { Impacted } \\
\text { Stream } \\
\text { Reach }\end{array}$} & \multirow{2}{*}{6} & 25.6 & 7.6 & 11.8 & 5.1 & 18.8 & 4.0 & 125.2 \\
\hline & & $( \pm 7.4)$ & $( \pm 3.6)$ & $( \pm 4.7)$ & $( \pm 1.7)$ & $( \pm 7.9)$ & $( \pm 2.3)$ & $( \pm 45.1)$ \\
\hline & \multirow[b]{2}{*}{7} & 23.1 & 6.7 & 8.4 & 4.0 & 13.5 & 2.6 & 123.0 \\
\hline & & $( \pm 5.5)$ & $( \pm 6.5)$ & $( \pm 2.6)$ & $( \pm 1.2)$ & $( \pm 3.3)$ & $( \pm 1.7)$ & $( \pm 30.1)$ \\
\hline & \multirow{2}{*}{8} & 21.3 & 6.5 & 6.4 & 3.8 & 13 & 1.9 & 128.1 \\
\hline & & $( \pm 4.7)$ & $( \pm 2.6)$ & $( \pm 1.5)$ & $( \pm 1.3)$ & $( \pm 3.2)$ & $( \pm 1.4)$ & $( \pm 30.7)$ \\
\hline \multirow{2}{*}{$\begin{array}{c}\text { Overall } \\
\text { mean }( \pm \text { SD })\end{array}$} & & 23.10 & 7.00 & 8.84 & 4.29 & 15.20 & 2.82 & 62.39 \\
\hline & & $( \pm 6.14)$ & $( \pm 3.27)$ & $( \pm 3.94)$ & $( \pm 1.54)$ & $( \pm 5.87)$ & $( \pm 2.02)$ & $( \pm 18.48)$ \\
\hline
\end{tabular}


Table 4. The overall mean concentrations of the investigated nutrient compounds and oxygen parameters of the receiving stream and effluent samples

\begin{tabular}{|c|c|c|c|c|c|c|c|c|c|c|}
\hline \multirow{2}{*}{ Waterbody } & \multirow{2}{*}{ : } & \multirow{2}{*}{$\begin{array}{c}\% \mathrm{O}_{2} \\
\text { Sat. }\end{array}$} & DO & BOD $_{5}$ & $\mathrm{NO}_{3}{ }^{-1}$ & $\mathrm{NO}_{2}$ & $\mathrm{NH}_{4}^{+}$ & $\mathrm{PO}_{4}{ }^{3-}$ & TOC & $\mathrm{SiO}_{2}$ \\
\hline & & & \multicolumn{8}{|c|}{$\left(\mathrm{mgl}^{-1}\right)$} \\
\hline \multirow{3}{*}{$\begin{array}{c}\text { Unimpacted } \\
\text { Stream } \\
\text { Reach }\end{array}$} & 1 & $\begin{array}{c}71.2 \\
( \pm 21.4)\end{array}$ & $\begin{array}{c}5.7 \\
( \pm 1.7)\end{array}$ & $\begin{array}{c}2.2 \\
( \pm 1.3)\end{array}$ & $\begin{array}{c}0.5 \\
( \pm 0.4)\end{array}$ & $\begin{array}{c}0.2 \\
( \pm 0.1)\end{array}$ & $\begin{array}{c}0.009 \\
( \pm 0.008)\end{array}$ & $\begin{array}{c}0.017 \\
( \pm 0.008)\end{array}$ & $\begin{array}{c}0.11 \\
( \pm 0.07)\end{array}$ & $\begin{array}{c}20.2 \\
( \pm 2.4)\end{array}$ \\
\hline & 2 & $\begin{array}{c}43.9 \\
( \pm 15.4)\end{array}$ & $\begin{array}{c}3.6 \\
( \pm 1.3)\end{array}$ & $\begin{array}{c}2.7 \\
( \pm 1.1)\end{array}$ & $\begin{array}{c}0.6 \\
( \pm 0.5)\end{array}$ & $\begin{array}{c}0.3 \\
\pm 0.1)\end{array}$ & $\begin{array}{c}0.065 \\
( \pm 0.05)\end{array}$ & $\begin{array}{c}0.026 \\
( \pm 0.017)\end{array}$ & $\begin{array}{c}0.15 \\
( \pm 0.08)\end{array}$ & $\begin{array}{c}18.6 \\
( \pm 1.3)\end{array}$ \\
\hline & 3 & $\begin{array}{c}76.4 \\
( \pm 11.6)\end{array}$ & $\begin{array}{c}6.2 \\
( \pm 0.9)\end{array}$ & $\begin{array}{c}2.7 \\
( \pm 1.3)\end{array}$ & $\begin{array}{c}0.9 \\
( \pm 0.5)\end{array}$ & $\begin{array}{c}0.2 \\
( \pm 0.1)\end{array}$ & $\begin{array}{c}0.057 \\
( \pm 0.062)\end{array}$ & $\begin{array}{c}0.026 \\
( \pm 0.016)\end{array}$ & $\begin{array}{c}0.11 \\
( \pm 0.06)\end{array}$ & $\begin{array}{c}17.8 \\
( \pm 1.2)\end{array}$ \\
\hline $\begin{array}{c}\text { Overall } \\
\text { mean }( \pm \text { SD) }\end{array}$ & & $\begin{array}{c}63.63 \\
( \pm 22.33)\end{array}$ & $\begin{array}{c}5.19 \\
( \pm 1.76)\end{array}$ & $\begin{array}{c}2.53 \\
( \pm 1.25)\end{array}$ & $\begin{array}{c}0.65 \\
( \pm 0.49)\end{array}$ & $\begin{array}{c}0.22 \\
( \pm 0.07)\end{array}$ & $\begin{array}{c}0.08 \\
( \pm 0.07)\end{array}$ & $\begin{array}{c}0.023 \\
( \pm 0.015)\end{array}$ & $\begin{array}{c}0.12 \\
( \pm 0.07)\end{array}$ & $\begin{array}{l}18.87 \\
( \pm 2.1)\end{array}$ \\
\hline \multirow{2}{*}{$\begin{array}{c}\text { Effluent } \\
\text { discharges }\end{array}$} & 4 & $\begin{array}{c}2.0 \\
( \pm 5.3)\end{array}$ & $\begin{array}{c}0.2 \\
( \pm 0.4)\end{array}$ & $\begin{array}{c}292.5 \\
\pm 218.3)\end{array}$ & $\begin{array}{c}13.2 \\
( \pm 2.7)\end{array}$ & $\begin{array}{c}2.3 \\
( \pm 0.8)\end{array}$ & $\begin{array}{c}0.298 \\
( \pm 0.15)\end{array}$ & $\begin{array}{c}2.384 \\
( \pm 1.676)\end{array}$ & $\begin{array}{c}1.84 \\
( \pm 1.27)\end{array}$ & $\begin{array}{c}20.3 \\
( \pm 2.2)\end{array}$ \\
\hline & 5 & $\begin{array}{c}27.9 \\
( \pm 26.7)\end{array}$ & $\begin{array}{c}2.2 \\
( \pm 2.1)\end{array}$ & $\begin{array}{l}270.1 \\
( \pm 217)\end{array}$ & $\begin{array}{c}13.2 \\
( \pm 3.8)\end{array}$ & $\begin{array}{c}2.6 \\
( \pm 1.0)\end{array}$ & $\begin{array}{c}0.275 \\
( \pm 0.091)\end{array}$ & $\begin{array}{c}2.729 \\
( \pm 2.149)\end{array}$ & $\begin{array}{c}1.32 \\
( \pm 1.1)\end{array}$ & $\begin{array}{c}18.8 \\
( \pm 1.8)\end{array}$ \\
\hline $\begin{array}{c}\text { Overall } \\
\text { mean }( \pm \text { SD) }\end{array}$ & & $\begin{array}{c}14.92 \\
( \pm 23.62)\end{array}$ & $\begin{array}{c}1.20 \\
( \pm 1.88)\end{array}$ & $\begin{array}{c}281.26 \\
( \pm 221.4)\end{array}$ & $\begin{array}{c}13.4 \\
( \pm 3.54)\end{array}$ & $\begin{array}{c}2.45 \\
( \pm 0.9)\end{array}$ & $\begin{array}{c}0.29 \\
( \pm 0.12)\end{array}$ & $\begin{array}{c}2.56 \\
\pm 1.96)\end{array}$ & $\begin{array}{c}1.58 \\
( \pm 1.24)\end{array}$ & $\begin{array}{l}440.0 \\
( \pm 187)\end{array}$ \\
\hline \multirow{3}{*}{$\begin{array}{c}\text { Impacted } \\
\text { Stream } \\
\text { Reach }\end{array}$} & 6 & $\begin{array}{c}21.4 \\
( \pm 18.1)\end{array}$ & $\begin{array}{c}1.8 \\
( \pm 1.5)\end{array}$ & $\begin{array}{c}77.1 \\
( \pm 54.7)\end{array}$ & $\begin{array}{c}2.1 \\
( \pm 0.9)\end{array}$ & $\begin{array}{c}0.4 \\
( \pm 0.2)\end{array}$ & $\begin{array}{l}0.189 \\
( \pm 0.1)\end{array}$ & $\begin{array}{c}0.22 \\
( \pm 0.164)\end{array}$ & $\begin{array}{c}0.39 \\
( \pm 0.56)\end{array}$ & $\begin{array}{c}19.6 \\
( \pm 1.3)\end{array}$ \\
\hline & 7 & $\begin{array}{c}43.1 \\
( \pm 22.6)\end{array}$ & $\begin{array}{c}3.7 \\
( \pm 2.2)\end{array}$ & $\begin{array}{c}42.1 \\
( \pm 45.9)\end{array}$ & $\begin{array}{c}1.2 \\
( \pm 0.5)\end{array}$ & $\begin{array}{c}0.3 \\
( \pm 0.1)\end{array}$ & $\begin{array}{c}0.103 \\
( \pm 0.065)\end{array}$ & $\begin{array}{c}0.058 \\
( \pm 0.065)\end{array}$ & $\begin{array}{c}0.18 \\
( \pm 0.07)\end{array}$ & $\begin{array}{c}18.1 \\
( \pm 1.4)\end{array}$ \\
\hline & 8 & $\begin{array}{c}34.1 \\
( \pm 22.6)\end{array}$ & $\begin{array}{c}2.8 \\
( \pm 1.9)\end{array}$ & $\begin{array}{c}37.6 \\
( \pm 44.5)\end{array}$ & $\begin{array}{c}0.9 \\
( \pm 0.5)\end{array}$ & $\begin{array}{c}0.2 \\
( \pm 0.1)\end{array}$ & $\begin{array}{c}0.049 \\
( \pm 0.034)\end{array}$ & $\begin{array}{c}0.049 \\
( \pm 0.034)\end{array}$ & $\begin{array}{c}0.17 \\
( \pm 0.08)\end{array}$ & $\begin{array}{c}17.9 \\
( \pm 1.3)\end{array}$ \\
\hline $\begin{array}{c}\text { Overall } \\
\text { mean }( \pm \mathrm{SD})\end{array}$ & & $\begin{array}{c}33.17 \\
( \pm 24.51)\end{array}$ & $\begin{array}{c}2.72 \\
( \pm 2.05)\end{array}$ & $\begin{array}{c}52.23 \\
( \pm 52.23)\end{array}$ & $\begin{array}{c}1.37 \\
( \pm 0.86)\end{array}$ & $\begin{array}{c}0.30 \\
( \pm 0.2)\end{array}$ & $\begin{array}{c}0.11 \\
( \pm 0.09)\end{array}$ & $\begin{array}{c}0.109 \\
( \pm 0.135)\end{array}$ & $\begin{array}{c}0.25 \\
( \pm 0.35)\end{array}$ & $\begin{array}{c}18.49 \\
( \pm 1.48)\end{array}$ \\
\hline
\end{tabular}

The $\mathrm{pH}$ of both the receiving stream and effluent sources varied from 6.6 to 7.3 (that is, from moderately acidic through neutral to moderately alkaline) as shown in Table 3 . The mean $\mathrm{pH}$ values recorded in this study is within the stipulated Nigerian FEPA pH tolerance limit of 6.0 - 9.0 for effluents to be discharged into surface water.

The recorded overall average concentrations of biological oxygen demand (BOD) in the unimpacted and impacted reach were $2.53 \mathrm{mgl}^{-1}$ and $52.23 \mathrm{mgl}^{-1}$ respectively while the overall average value recorded for the effluent sources was $281.26 \mathrm{mgl}^{-1}$ as shown in Table 4. Willms and Pelletier (1992) recorded $316 \mathrm{mgl}^{-1} \mathrm{BOD}$ in the influent stream into Eastern State hospital wastewater treatment plant in USA, while Emmanuel et al. (2001) recorded an average of $603 \mathrm{mgl}^{-1} \mathrm{BOD}$ in a hospital effluent stream in France. In the present study, BOD in OAUTHC effluent occurred in the range of $54.4-680 \mathrm{mgl}^{-1}$ in the sewer effluent (station 4) and $5.10-645$ $\mathrm{mgl}^{-1}$ in the open gutter effluent stream (station 5), and based on the Metcalf and Eddy (1991) classification, the wastewater effluents in this study can therefore be classified as being between medium and strong strength wastewater. The overall average recorded $\mathrm{BOD}_{5}$ concentration in the receiving stream and effluents sources are higher than the $50 \mathrm{mgl}^{-1}$ tolerance level stipulated by FEPA for effluents to be discharged into the surface water and this is very unfortunate since the continuous discharge of the effluent has seriously impacted the receiving stream and this may have negative effects on the quality of the freshwater and can as well cause harm to the aquatic life especially downstream (Ogunfowokan et al., 2005). Eludoyin et al. (2004) arrived at a similar conclusion studying the effect of Oja-titun market effluent on the chemical quality of the receiving Opa reservoir in Ile-Ife, Nigeria, that the discharge of effluents with high BOD loading into fresh water bodies may give rise to organic pollution.

Table 5 shows the comparison of the overall mean concentrations of all the investigated physico-chemical parameters in both the unimpacted and impacted stream reach of the receiving stream The difference between the water quality of unimpacted and impacted receiving water bodies was significant $(\mathrm{P} \leq 0.05)$ for almost the investigated parameters. The table shows $\mathrm{SO}_{4}{ }^{2-}, \mathrm{PO}_{4}{ }^{3-}$ and $\mathrm{BOD}_{5}$ to be more than 3 orders of magnitude greater in the impacted than in the unimpacted stream reach. 
Table 5. Relationship between the water quality parameters of unimpacted and impacted reach of the receiving stream

\begin{tabular}{|c|c|c|c|c|}
\hline \multirow{2}{*}{ Parameter } & $\begin{array}{c}\text { Unimpacted } \\
\text { Stream Reach } \\
\text { (USR) }\end{array}$ & $\begin{array}{l}\text { Impacted Stream } \\
\text { Reach (ISR) }\end{array}$ & \multirow[b]{2}{*}{$\mathbf{X}_{\text {ISR }} / \mathbf{X}_{\text {USR }}$} & \multirow[b]{2}{*}{ P Value } \\
\hline & $\begin{array}{l}\text { X USR } \\
(n=48)\end{array}$ & $\begin{array}{l}\text { X ISR } \\
(n=48)\end{array}$ & & \\
\hline \multicolumn{5}{|l|}{ Hydro - Physical } \\
\hline Water Temp. $\left({ }^{\circ} \mathrm{C}\right)$ & $25.49( \pm 1.97)$ & $25.00( \pm 1.64)$ & 0.98 & 0.366 \\
\hline Colour (Pt.Co.) & $86.58( \pm 53.91)$ & $113.58( \pm 70.99)$ & 1.31 & 0.000 \\
\hline Turbidity (NTU) & $20.60( \pm 12.35)$ & $29.04( \pm 15.94)$ & 1.41 & 0.000 \\
\hline $\operatorname{TSS} \quad\left(\mathrm{mgl}^{-1}\right)$ & $11.0( \pm 7.0)$ & $26.0( \pm 14.0)$ & 2.36 & 0.000 \\
\hline \multicolumn{5}{|l|}{ General Chemical } \\
\hline $\mathrm{pH}$ & $6.88( \pm 0.24)$ & $7.0( \pm 0.20)$ & 1.02 & 0.000 \\
\hline Cond. $(\mu \mathrm{S} / \mathrm{cm})$ & $135.63( \pm 25.6)$ & $219.81( \pm 67.28)$ & 1.62 & 0.004 \\
\hline TDS $\left(\mathrm{mgl}^{-1}\right)$ & $96.52( \pm 16.38)$ & $150.56( \pm 44.57)$ & 1.56 & 0.002 \\
\hline $\mathrm{TS} \quad\left(\mathrm{mgl}^{-1}\right)$ & $117.56( \pm 24.92)$ & $191.56( \pm 54.5)$ & 1.63 & 0.000 \\
\hline Alka. $\left(\mathrm{mgCaCO}_{3} 1^{-1}\right)$ & $63.29( \pm 13.19)$ & $104.31( \pm 30.93)$ & 1.65 & 0.001 \\
\hline $\operatorname{Acidity}\left(\mathrm{mgCaCO}_{3} \mathrm{l}^{-1}\right)$ & $19.47( \pm 8.19)$ & $28.35( \pm 17.12)$ & 1.46 & 0.001 \\
\hline \multicolumn{5}{|l|}{ Major Ions $\left(\mathrm{mgl}^{-1}\right)$} \\
\hline $\mathrm{Ca}^{2+}$ & $14.57( \pm 2.62)$ & $23.10( \pm 6.14)$ & 1.58 & 0.000 \\
\hline $\mathrm{Mg}^{2+}$ & $4.92( \pm 1.60)$ & $7.00( \pm 3.27)$ & 1.42 & 0.093 \\
\hline $\mathrm{Na}^{+}$ & $4.82( \pm 1.4)$ & $8.84( \pm 3.94)$ & 1.83 & 0.000 \\
\hline $\mathrm{K}^{+}$ & $2.64( \pm 0.68)$ & $4.29( \pm 1.54)$ & 1.62 & 0.000 \\
\hline $\mathrm{Cl}^{-}$ & $10.74( \pm 1.71)$ & $15.20( \pm 5.87)$ & 1.42 & 0.001 \\
\hline $\mathrm{SO}_{4}^{2-}$ & $0.80( \pm 0.65)$ & $2.82( \pm 2.02)$ & 3.52 & 0.000 \\
\hline $\mathrm{HCO}_{3}^{-}$ & $14.57( \pm 2.62)$ & $23.10( \pm 6.14)$ & 1.64 & 0.000 \\
\hline \multicolumn{5}{|l|}{$\begin{array}{l}\text { Nutrients and oxygen } \\
\text { Compound }\end{array}$} \\
\hline$\% \mathrm{O}_{2}$ Sat. & $63.63( \pm 22.33)$ & $33.17( \pm 24.51)$ & 0.52 & 0.000 \\
\hline $\mathrm{DO}\left(\mathrm{mgl}^{-1}\right)$ & $5.19( \pm 1.76)$ & $2.72( \pm 2.05)$ & 0.52 & 0.000 \\
\hline $\mathrm{BOD}_{5}\left(\mathrm{mgl}^{-1}\right)$ & $2.53( \pm 1.25)$ & $52.23( \pm 52.23)$ & 20.64 & 0.000 \\
\hline $\mathrm{NO}_{3}^{-}\left(\mathrm{mgl}^{-1}\right)$ & $0.65( \pm 0.49)$ & $1.37( \pm 0.86)$ & 2.11 & 0.000 \\
\hline $\mathrm{NO}_{2}^{-}\left(\mathrm{mgl}^{-1}\right)$ & $0.22( \pm 0.07)$ & $0.30( \pm 0.2)$ & 1.36 & 0.000 \\
\hline $\mathrm{NH}_{4}^{+}\left(\mathrm{mgl}^{-1}\right)$ & $0.08( \pm 0.07)$ & $0.11( \pm 0.09)$ & 1.38 & 0.000 \\
\hline $\mathrm{PO}_{4}^{3-}\left(\mathrm{mgl}^{-1}\right)$ & $0.023( \pm 0.015)$ & $0.109( \pm 0.135)$ & 4.74 & 0.000 \\
\hline TOC $\left(\mathrm{mgl}^{-1}\right)$ & $0.12( \pm 0.07)$ & $0.25( \pm 0.35)$ & 2.08 & 0.000 \\
\hline $\mathrm{SiO}_{2}\left(\mathrm{mgl}^{-1}\right)$ & $18.87( \pm 2.1)$ & $18.49( \pm 1.48)$ & 0.98 & 0.000 \\
\hline
\end{tabular}




\subsection{Effluent Pollution Impact and Stream Recovery}

Table 6 shows the percentage impact of the wastewater effluent discharged into the receiving stream at stations 6 and 8 from OAUTHC two pollution point sources, and the extent to which the stream has recovered at the last investigated station 8 . The observed trend of stream recovery from pollution impact in this study shows a general progressive increases from station 7 to the last investigated station 8 , and this trend follows the stream recovery pattern described by Nathanson (2000) and Peirce et al. (1997). However the increase pH and reduction in dissolved oxygen (DO) observed especially in station 8 are traceable to the fact the station is characterized by reduced stream velocity and eutrophication tendency.

Table 6. Effluent discharge Impact on water quality parameters of the receiving stream and recovery

\begin{tabular}{|c|c|c|c|}
\hline Parameter & $\begin{array}{l}\text { \% Impact at } \\
\text { station } 6\end{array}$ & $\begin{array}{c}\text { \% Impact at station } \\
8\end{array}$ & $\begin{array}{c}\% \text { Recovery at Station } \\
8\end{array}$ \\
\hline \multicolumn{4}{|l|}{ Hydro- Physical } \\
\hline Water Temp. $\left({ }^{\circ} \mathrm{C}\right)$ & 1.55 & 2.37 & -34.43 \\
\hline Colour (Pt.Co.) & 51.71 & 34.86 & 48.34 \\
\hline Turbidity (NTU) & 52.20 & 17.05 & 206.26 \\
\hline $\operatorname{TSS}\left(\mathrm{mgl}^{-1}\right)$ & 66.33 & 22.73 & 191.83 \\
\hline \multicolumn{4}{|l|}{ General Chemical } \\
\hline $\mathrm{pH}$ & 0.00 & 2.27 & -100.00 \\
\hline Cond. $(\mu \mathrm{S} / \mathrm{cm})$ & 42.53 & 36.02 & 18.06 \\
\hline $\operatorname{TDS}\left(\mathrm{mgl}^{-1}\right)$ & 42.75 & 29.85 & 43.20 \\
\hline $\mathrm{TS}\left(\mathrm{mgl}^{-1}\right)$ & 49.06 & 28.62 & 71.42 \\
\hline Alka. $\left(\mathrm{mgCaCO}_{3} 1^{-1}\right)$ & 44.87 & 33.81 & 32.71 \\
\hline $\operatorname{Acidity}\left(\mathrm{mgCaCO}_{3} 1^{-1}\right)$ & 44.69 & 21.17 & 111.05 \\
\hline \multicolumn{4}{|l|}{ Major Ions (mgl $\left.{ }^{-1}\right)$} \\
\hline $\mathrm{Ca}^{2+}$ & 43.09 & 31.60 & 36.36 \\
\hline $\mathrm{Mg}^{2+}$ & 35.26 & 24.31 & 45.07 \\
\hline $\mathrm{Na}^{+}$ & 59.15 & 24.69 & 139.61 \\
\hline $\mathrm{K}^{+}$ & 48.24 & 30.53 & 58.01 \\
\hline $\mathrm{Cl}^{-}$ & 42.87 & 17.38 & 146.61 \\
\hline $\mathrm{SO}_{4}^{2-}$ & 80.00 & 57.89 & 38.18 \\
\hline $\mathrm{HCO}_{3}^{-}$ & 69.54 & 70.23 & -0.98 \\
\hline \multicolumn{4}{|c|}{ Nutrients and Oxygen Compound } \\
\hline $\mathrm{DO}\left(\mathrm{mgl}^{-1}\right)$ & 197.34 & 86.60 & 127.88 \\
\hline$\% \mathrm{O}_{2}$ Sat. & 188.33 & 85.36 & 120.64 \\
\hline $\mathrm{BOD}_{5}\left(\mathrm{mgl}^{-1}\right)$ & 96.72 & 93.27 & 3.70 \\
\hline $\mathrm{NO}_{3}^{-}\left(\mathrm{mgl}^{-1}\right)$ & 69.05 & 27.78 & 148.57 \\
\hline $\mathrm{NO}_{2}^{-}\left(\mathrm{mgl}^{-1}\right)$ & 45.00 & 10.00 & 350.00 \\
\hline $\mathrm{NH}_{4}^{+}\left(\mathrm{mgl}^{-1}\right)$ & 57.67 & 63.27 & -8.84 \\
\hline $\mathrm{PO}_{4}^{3-}\left(\mathrm{mgl}^{-1}\right)$ & 89.55 & 53.06 & 68.76 \\
\hline $\mathrm{TOC}\left(\mathrm{mgl}^{-1}\right)$ & 69.23 & 29.41 & 135.38 \\
\hline $\mathrm{SiO}_{2}\left(\mathrm{mgl}^{-1}\right)$ & 3.72 & 5.42 & -31.27 \\
\hline
\end{tabular}




\section{Conclusion}

This study characterized the physico-chemical quality of the two wastewater point sources from the Obafemi Awolowo University Teaching Hospitals Complex (OAUTHC) Ile-Ife, and assessed their impacts on the water quality of the receiving Elekete stream. The water qualities from the unimpacted section of the receiving Elekete stream meet most of the general water requirements for both domestic and industrial uses. The investigated physico-chemical quality showed that $\mathrm{BOD}_{5}$ and TSS of effluents from OAUTHC discharge points were above the FEPA (1991) effluent limitation standards. The effluents quality varied widely, and decreased gradually from the effluent outfall downstream. The difference between the water quality of unimpacted and impacted receiving water bodies was significant $(\mathrm{P} \leq 0.05)$ for most of the parameters (except water temperature and magnesium) as most of the investigated parameters were higher in the impacted section of the stream, thus indicating an impact on the receiving stream. The pronounced increase in the $\mathrm{BOD}_{5}$ values of the impacted section of receiving stream indicated that the discharge from Obafemi Awolowo University Teaching Hospitals Complex Ile-Ife was mainly organic in nature. It is therefore recommended for OAUTHC authority to design and put in place an appropriate wastewater treatment plant within the hospital facility.

\section{Acknowledgement}

The authors wish to acknowledge the assistance received from Mr. Adesuji Adejuwon and Miss Dinat Dutze during the collection and analyses of the samples. We also thank Dr O.J. Owojori of the Department of Zoology, Obafemi Awolowo University, Ile-Ife, Nigeria for invaluable suggestions on earlier drafts of the manuscript.

\section{References}

APHA, AWWA, \& WEF (1995). Standard methods for the examination of water and wastewater (19th, ed., p. 904). Washington.

CCLIN Paris-Nord. (1999). Élimination des effluents liqiudes des établissements hospitaliers Recommandations (p. 74). Paris: Instut Biomédical des Cordeliers.

Chapman, D. E. (1992). Water Quality Assessments: A guide to the use of biota, sediments and water in environmental monitoring (p. 585). UNESCO / WHO / UNEP, Champman and Hall, London.

De lange, E. (1994). Manual for simple water quality analysis (pp. 6.1-6.13). International Water Treatment Foundation. Amsterdam.

Deloffre-Bonnamour, N. (1995). Les rejets des établissements de santé: des effluents liqiudes aux déchets solides (p. 72). Mémoire de Maîtrise, Université Claude Bernard-Lyon1, Institut Universitaire Professionnalisé, Génie de l'Environnement-Ecodéveloppement, Lyon.

DPR. (1991). Environmental guidelines and standards for the petroleum industry in Nigeria (p. 171). Department of Petroleum Resources (DPR), Lagos.

Eludoyin, A. O., Ofoezie, I. E., \& Ogunkoya, O. O. (2004). The effect of Oja-titun market effluent on the chemical quality of receiving Opa reservoir in Ile-Ife, Nigeria. Journal of Environmental Management, 72, 249 - 259. http://dx.doi.org/10.1016/j.jenvman.2004.05.005

Emmanuel, E., Blanchard, J. M., Keck, G., \& Perrodin, Y. (2001). Caractérisation chimique, biologique et écotoxicologique des effluents hospitaliers (pp. 31-33). Déchets Sciences et Techniques, revue francophone d'ecologie industrielle, N 22 - 2éme trimester.

FEPA. (1991). Guidelines and Standards for Environmental Control in Nigeria (p. 238). Federal Environmental Protection Agency (FEPA). Government Press. Lagos.

Gautam, A. K., Kumar, S., \& Sabumon, P. C. (2007). Preliminary study of physico-chemical treatment options for hospital wastewater. Journal of Environmental Management, 83, 298 - 306. http://dx.doi.org/10.1016/j.jenvman.2006.03.009

Golterman, H. L., Clymo, R. S., \& Ohnstade, M. A. M. (1978). Methods for Physical and Chemical Analysis of Freshwaters (2nd ed., p. 215). Blackwell Scientific Publications. London.

Hellawell, J. M. (1986). Biological indicator of freshwater pollution and environmental management (p. 509). Elservier Science Publishers, England. http://dx.doi.org/10.1007/978-94-009-4315-5_2

Jackson, M. K., Morris, G. P., Smith, P. G., \& Crawford, J. F. (1989). Environmental Health Reference Book (pp. 10.1-10.34). London: Butterworth - Heinemann.

Jehannin, P. (1999). Caractérisation et gestion des rejets liquides hospitaliers - Etude partculiére de la situation 
du C.H. de Hyéres (Var) (p. 100). Mémoire de fin d'études, Ecole Nationale de la Santé Publique, Rennes.

Metcalf, \& Eddy. (1991). Wastewater Engineering (3rd ed., p. 1334). New York: McGraw-Hill.

Montague, P. (1998). Drug in the water. Rachel's Environment \& Health Weekly 614. Environmental Research Foundation, USA.

Nathanson, J. A. (2000). Basic Environmental Technology: water supply, waste management and pollution control (3rd ed., p. 496). Prentice-Hall Inc. USA.

NSFC. (1996). On- site Wastewater Disposal and Public Health. Pipeline, summer 1996, Vol.7, N0.3. National Small Flow Clearinghouse (NSFC).

OAUTHC Newsletter. (2001). Newsletter Vol.17 No.1, April - June .Obafemi Awolowo University Teaching Hospitals Complex (OAUTHC).

Ogunfowokan, O. A., Okoh, E. K., Adenuga, A. A., \& Asubiojo, O. I. (2005). An Assessment of the impact of point source polluton from a university sewage treatment oxidation pond on a receiving stream- a preliminary study. Journal of Applied Sciences, 5(1), 36 - 43. http://dx.doi.org/10.3923/jas.2005.36.43

Peirce, J. J., Weiner, R. F., \& Vesilind, P. A. (1990). Environmental Pollution and Control (4th ed., p. 393). Butterworth - Heinemann, USA.

Raloff, J. (1998). Drugged Waters. Science News, 153(12), 187 - 189. http://dx.doi.org/10.2307/4010314

Stan, H. J., \& Linkerhäger, M. (1994). Occurrence of clofibric acid in aquatic system - Does the medical application cause a contamination of surface, ground and drinking water. Vom Wasser, 83, 57 - 68.

USEPA. (1983). Methods for chemical analysis of water and wastes. EMSL Cincinnnati OH 45268: US Environmental Protection Agency (USEPA).

WHO. (1985). Management of Waste from Hospitals and other Health Care Establishments. Report on a WHO meeting, Bergen, 28 June -1 July 1983.Copenhagen, World Health Organization (WHO) Regional office for Europe. (Euro Reports and Studies, No. 97).

Willms, R., \& Pelletier, G. (1992). Impacts of Eastern State Hospital and Lakeland Village Wastewater Discharges on the Quality of West Medical Lake (p. 36). Washington State Department of Ecology, Watershed Section, Olympia, Washington 98504 - 7710.Publication No. 92 - e63. 